\title{
Transposition
}

Musique et Sciences Sociales

Hors-série 1 | 2018

Musique, histoire, sociétés

\section{Le « tournant » des années 1980 en \\ « Ethnomusicologie de la France »}

Retour sur un séminaire EHESS (1985-1993)

Jacques Cheyronnaud

\section{OpenEdition}

\section{Journals}

Édition électronique

URL : http://journals.openedition.org/transposition/1691

DOI : 10.4000/transposition. 1691

ISSN : 2110-6134

Éditeur

CRAL - Centre de recherche sur les arts et le langage

Référence électronique

Jacques Cheyronnaud, «Le « tournant » des années 1980 en « Ethnomusicologie de la France » »,

Transposition [En ligne], Hors-série 1 | 2018, mis en ligne le 30 janvier 2018, consulté le 11 février 2020.

URL : http://journals.openedition.org/transposition/1691; DOI : 10.4000/transposition.1691

Ce document a été généré automatiquement le 11 février 2020.

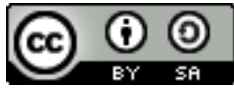

La revue Transposition est mise à disposition selon les termes de la Licence Creative Commons Attribution - Partage dans les Mêmes Conditions 4.0 International. 


\title{
Le « tournant » des années 1980 en « Ethnomusicologie de la France »
}

\author{
Retour sur un séminaire EHESS (1985-1993)
}

\author{
Jacques Cheyronnaud
}

La configuration programmatique qui prend le nom d'ethnomusicologie vers le milieu des années 1950, initiée en France dans la structure de musées d'ethnographie à partir des années 30 (Musée de l'Homme, 1929; Musée des Arts et Traditions populaires, 1937) bénéficia à partir de 1961 de l'opportunité académique que lui offrit la $\mathrm{VI}^{\mathrm{e}}$ Section de 1 'École Pratique des Hautes Études qui deviendrait l'École des Hautes études en Sciences Sociales (Ehess) en 1975 : une Charge de conférences complémentaires, qui fut confiée à Claudie Marcel-Dubois (1913-1989) ${ }^{1}$ dans le cadre de la direction d'études de Claude Lévi-Strauss. Ce séminaire, parallèlement à quelques autres formes non moins provisoires d'enseignement universitaire en la matière dans ces années 1960 constituera durablement un espace unique en France de traitements sur différentes aires culturelles, de thématiques généralement annuelles, auquel participaient régulièrement comme auditeurs ou intervenants ce que la spécialité comptait alors de chercheurs institutionnels et d'étudiants. Il se déroulera durant près de vingt ans, jusqu'à la fin de la décennie 1970. À cette époque, son intérêt s'était quelque peu émoussé et l'auditoire raréfié, les réseaux d'échanges collectifs et de rencontres en ethnomusicologie générale ou régionale et les projets de connaissance en la matière s'étant consolidés voire transformés au long des deux décennies. L'évolution de la démographie de la spécialité ne serait alors plus seulement due aux institutions muséales fondatrices qui en avaient longtemps sinon monopolisé du moins dominé l'exercice professionnel. Ajoutons dès ici, s'agissant du moins du Musée national des Arts et Traditions populaires (Mnatp) à la fin des mêmes années 1970 qu'une réflexion anthropologique autour d'une ethnologie des «sociétés complexes ${ }^{2}$ s'interrogeait sur l'avenir d'une tradition ethnologique que campait historiquement ce musée, et qui s'était abondamment vouée, comme en ethnomusicologie, à inscrire sous l'étiquette «musiques traditionnelles » les reliquats d'un ordre culturel trop facilement délimité par restrictions: non écrit, non savant, non urbain, non institutionnel, non 
contemporain; en somme, une ethnomusicologie régionale (" de la France»), inventoriale.

2 Aucun professionnel de l'ethnomusicologie ne s'est porté candidat à une reprise de la charge abandonnée par Claudie Marcel-Dubois. Celle qu'obtiendra peu après MarieMarguerite Pichonnet-Andral (Maguy Andral, 1922-2004), enseignement qui subsistera jusqu'en 1985 comme séminaire de département, ne revendiquait aucune continuité directe avec l'enseignement précédent. Chercheuse au CNRS, ancienne assistante depuis 1945 puis successeur en 1981 de C. Marcel-Dubois à la direction du Département d'ethnomusicologie-Phonothèque du Mnatp, Maguy Andral encadra un enseignement régional délimité au terrain hexagonal, éventuellement exceptionnellement élargi à l'Europe ${ }^{3}$. L'auditoire était constitué pour l'essentiel d'étudiants de l'EHESS inscrits sous sa direction (DEA, thèse) en ethnologie sur un objet « d'ethnomusicologie de la France » et d'auditeurs libres, le plus souvent boursiers de la Mission du Patrimoine ethnologique. Un enseignement spécifique au DEA Anthropologie sociale et Ethnologie de l'Ehess me fut confié en 1985 à la suite d'un programme que l'on m'avait suggéré de proposer ${ }^{4}$. Cet enseignement se tiendra de 1985 à 1993. L'occasion qui m'est offerte de présenter rétrospectivement le contenu de ce séminaire me permettra de lever d'éventuels malentendus qui consisteraient à décréter une continuité avec les enseignements précédents sous le label ou le prétexte d'une "ethnomusicologie de la France ». Il importe de préciser d'emblée le contexte de l'époque.

\section{Déplacement organisationnel}

3 Le paysage organisationnel de l'ethnomusicologie, longtemps dominé en France par deux sièges parisiens ancrés dans l'institution muséale (Musée de l'Homme, Mnatp) qui se partageaient volontiers, pas nécessairement de manière irénique, l'intitulé de « département ${ }^{5}$ » pour se désigner en unités programmatiques, allait connaître une profonde recomposition dans la traversée des années 1980. On ne traitera ici que du Mnatp et de son Département d'ethnomusicologie - Phonothèque.

4 Le début des années 80 serait fut marqué par le départ en retraite de CL. Marcel-Dubois et la prise en charge du Département par M. Andral. Cette succession modifierait sensiblement la forme de gestion qui avait cours précédemment. Derrière l'apparente unité programmatique qui avait prévalu jusqu'ici à travers la figure tutélaire de la fondatrice, une réalité intellectuelle plus complexe allait s'émanciper sur fond de « crises ». En premier lieu, sur fond d'une lancinante remise en question de l'institution même du "musée-laboratoire", unité administrative et programmatique alliant conservation et recherche qui articulait le Mnatp depuis sa fondation sur la colline de Chaillot. Était également concerné, dans cette remise en question de l'institution, le type d'ethnologie qu'elle représentait du moins historiquement, le plus souvent ordonné à la constitution de collections et à l'édition de corpus par régions de France (habitat, mobilier, costumes, etc.) auxquels l'on postposait communément la spécification «traditionnel». De nombreuses et vives critiques tant en interne qu'à l'extérieur du Musée s'étendaient expressément à ce "département» tel qu'il s'était historiquement configuré, tout à la fois voire indifféremment espace de science (programmes d'inventaire, méthodes, décisions de corpus et savoirs attachés à la classe d'objets dite "musique(s) de tradition orale») et lieu de conservation: une phonothèque, instituée depuis 1960 en service de documentation sonore, 
théoriquement dissocié des programmes précédents et ouvert à la conservation d'autres archives sonores que les musicales. Dans les faits, les opérations de l'un et l'autre s'enchevêtreront sur un même périmètre jusqu'à constituer une enclave passablement repliée sur elle-même. Quel avenir, se demandait-on à la fin des années 70, pour ce site enclavé qui campait une ethnomusicologie régionale qui s'était abondamment vouée à la confection de collections pour la plupart inaccessibles au public, en déficit flagrant de conservateurs et de chercheurs professionnels et dont les résultats tangibles comme par exemple la publication d'un corpus des "Musiques orales de France » ou la diffusion phonographique des collections étaient à peu près inexistants?

5 Le volontarisme politique du début des années 80 en matière de "réhabilitation culturelle » modifierait sensiblement le paysage de l'ethnomusicologie - c'est à ce titre que je parlerai ici de «tournant 1980 »-, notamment en se centrant sur la même classe d'objets que privilégiait, et sur laquelle théorisait l'ethnomusicologie régionale au Mnatp. L'administration de la musique, bientôt rejointe par la Mission du Patrimoine

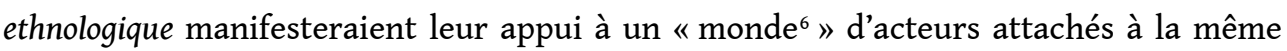
classe d'objets que cette ethnomusicologie inventoriale «de la France». Le volontarisme se dirigea également vers une confortation disciplinaire en université, en instaurant progressivement un cursus de spécialisation en ethnomusicologie (notamment à l'Université Paris X). À ces initiatives, s'ajouterait l'inscription dans les conservatoires et les écoles de musique, éventuellement en marge d'une conception «grammaticale» de la compétence de musicien ${ }^{7}$, d'enseignements de pratiques attachées à la classe des "musiques traditionnelles» de France ou d'autres aires culturelles. Ainsi, le paysage longtemps dominé institutionnellement par l'ethnomusicologie et ses sièges muséaux se voyait-il élargi sinon bousculé, au moins dans le cas régional de la France, par l'émergence de nouvelles catégories d'acteurs susceptibles de faire d'un intérêt pour la même classe d'objets que ceux de l'ethnomusicologie régionale, une possibilité de professionnalisation(s), du moins d'emplois dans les « métiers de la musique ».

6 La jeune Mission du Patrimoine ethnologique, créée en 1980 «Année du patrimoine » et dont des programmes se tiendraient à proximité de ceux du Mnatp prit une place non négligeable dans la dynamique évoquée ici. Elle n'entendait pas s'occuper précisément d'ethnomusicologie ou concurrencer l'administration de la musique en ce domaine. Les deux institutions pourront toutefois se croiser (non sans difficultés) au long de la décennie sur des objectifs d'appui aux organisations associatives œuvrant à la collecte et à la documentation de la classe des «musiques traditionnelles. Mais là où l'une parlait immédiatement d'ethnomusicologie et de corpus, l'autre évoquait méthodes d'enquêtes, de collecte, ethnographie ou ethnologie, voire réflexion sur quelques évidences comme celles de "tradition» ou de "société traditionnelle ${ }^{8}$ ». Les deux dispenseraient des bourses en direction de membres d'associations candidats à une formation.

7 C'est dans ce contexte que me sera suggéré en 1985 de présenter le projet d'enseignement et de formation qui se tiendrait dans le cadre de la Formation à la recherche en Anthropologie sociale et Ethnologie de l'Ehess. Cet enseignement intitulé Ethnologie de la musique - ethnomusicologie ${ }^{9}$ sera ouvert la même année; il accueillera durant huit années consécutives plusieurs dizaines d'auditeurs, étudiants du DEA Anthropologie sociale et Ethnologie, élèves candidats au diplôme de l'Ehess au sein de 
diverses directions d'études et, sur dossier, des auditeurs libres, pour la plupart boursiers de la Mission ou membres d'organisations associatives alors participant de leur propre initiative. Un double objectif avait été assigné au séminaire. Celui d'une part, de constituer un espace de réflexion ouvert tant à des musiciens, musicologues qu'à des étudiants non spécialistes mais éventuellement confrontés à un moment ou un autre dans leur projet de thèse en ethnologie de la France ou d'autres régions culturelles, à des questions d'ordre musical. Le choix des thèmes serait guidé par cet autre objectif: accueillir des auditeurs issus d'organisations associatives, leur permettre de formuler une problématique de travail concernant le secteur des musiques traditionnelles, questionner des présupposés, offrir des perspectives éventuellement jusqu'à la préparation du diplôme de l'École qui permettrait ainsi d'intégrer un cursus universitaire ou de "valider » un parcours spécialisé. L'objectif était alors que la relation de connaissance et d'attachement, l'engagement de sensibilité que portaient les personnes à «la musique traditionnelle " (pratiques, répertoires, instruments de musique, etc.) fassent l'objet de l'épreuve de communication publique dans un contexte universitaire et de recherche où la recevabilité imposait des critères de méthode et de précision, de rigueur et de probité. Cette exigence passait par la constitution d'un espace propre de réflexivité critique concernant le choix de l'objet d'étude, les méthodes, les concepts ou les notions.

\section{Geste patrimoniale ethno-musicographique}

8 Plusieurs problématiques solidaires entre elles, choisies parce que susceptibles d'intéresser les profils assez diversifiés des participants furent abordées de manière plus ou moins récurrente durant les huit années de séminaire. L'une d'elles concernait quelques opérations de confection de grands corpus « hymnodiques» en France et en Europe dans la traversée du $\mathrm{xIX}^{\mathrm{e}}$ siècle $^{10}$, ce qui permettait de s'attarder sur le mouvement d'élaboration savante d'une classe de répertoires et de pratiques sous l'appellation générique de "chants et chansons populaires ». Cette problématique en croisait délibérément une autre, musicologique et anthropologique, tenant à une relecture de la mise au point progressive au cours des siècles de notre système graphique de notation musicale à partir des premiers essais neumatiques ${ }^{11}$. Un aperçu historique des formes procédurales de «délocalisation» (les techniques de déplacement d'une émission vocale ou instrumentale de sa source de production) permettait ainsi de poser un regard sur la mise au point, dans nos sociétés, de normes d'individuation et des solutions fiables de maîtrise du déplacement, du système de notation musicale qui prévalait dans nos sociétés jusqu'à l'enregistrement sonore. Cette problématique servait aussi de cadre général à une revisite de grandes enquêtes et collectes en France concernant la classe des "musiques traditionnelles », les normes méthodologiques, les modes procéduraux autour desquels se cristallisait une "dramatique de l'arraisonnement ${ }^{12}$ ». Cette dramatique se donnait de triompher d'une double perte : celle avancée par le folklorisme, d'une « culture populaire » moribonde ; celle d'énoncés «chants et chansons" proposés par ce même folklorisme, indissociables de la présence physique qui se dépensait dans leur énonciation et exigeait la mobilisation de ressources d'interception ${ }^{13}$.

9 Le thème serait alors celui d'une "geste " d'arraisonnement: " geste patrimoniale ethno-musicographique» (désormais Geste) qui n'avait de cesse de plaider depuis le 
XIX ${ }^{e}$ siècle l'urgence, la perte ou la fin, voire la grandeur d'un dévouement d'investigation, qui en appelait à la collecte et à la conservation sous les mots d'ordre de sauvegarde ou de sauvetage. Moments d'intensité, formes d'aboutissements, péripéties, opportunités, continuités, déplacements ou ruptures ponctuaient l'itinéraire de cette Geste, jusqu'à l'avènement d'institutions spécialisées. Elle se réalisait en un dispositif méthodologique avançant ses attendus, ses objectifs, fixant ou déterminant l'autorité des objets de sa quête. Ainsi d'une vaste littérature procédurale d'instructions, de questionnaires chargés de configurer une individuation de ses objets pour les délocaliser et les affilier, en leur déterminant une définition, en spécifiant ce qui doit avoir trait à leur identification (en quoi ils devraient offrir une pertinence, une exemplarité). Le projet d'un Recueil général des poésies populaires de la France initié en septembre 1852, la rédaction et la diffusion de ses Instructions constitueraient à coup sûr un moment d'intensité de l'itinéraire ${ }^{14}$. Il se poursuivrait en bien d'autres ${ }^{15}$. L'entrée, à l'articulation des $\mathrm{XIX}^{\mathrm{e}}$ et $\mathrm{Xx}^{\mathrm{e}}$ siècles et à proximité des folkloristes, de musiciens-collecteurs d'une "véritable chanson populaire » autour de Charles Bordes et de la Schola Cantorum - des musiciens et musicologues du régionalisme, leur idéal politique et culturel si ce n'est moral de « régénération » d'une « musique française » constituerait un autre moment d'intensité, alors voué à la célébration musicale des objets d'une Geste largement dominée jusqu'ici par une célébration littéraire facilement condescendante d'une " poésie populaire ».

10 L'avènement de l'enregistrement sonore à l'articulation des deux siècles viendrait offrir une opportunité de poids et une ressource de plus en plus incontournable pour l'entreprise patrimoniale, bouleversant l'économie d'interception et de transfert jusqu'alors centrée sur l'arraisonnement graphique. L'histoire de ce que l'on a pu appeler des «incunables du son» est aujourd'hui bien connue. À l'instar de pays européens, régulièrement mentionnés dans les histoires de l'ethnomusicologie proposées par les fondateurs de ce secteur en France, des institutions vont peu à peu s'ériger en centres de programmes, d'inventaire et de capitalisation patrimoniale. Le mode d'inscription "aurale» (finalisé pour l'oreille et l'entendre) sur supports magnétiques ouvrant sur l'écoute à distance comme en laboratoire, deviendra une obsession méthodologique? Le regard rétrospectif sur la Geste que proposait le séminaire suggérait alors d'inscrire le Mnatp au nombre de ses moments d'intensité dans la contemporanéité, non sans déplacements ou ruptures, voire péripéties. Les fondateurs musicologues du Mnatp convoquaient des auteurs théoriciens, historiens et savants de la «chanson populaire » des courants précédents parmi leurs précurseurs, pour signifier tout à la fois grandeur et permanence de la dynamique d'intérêt pour des répertoires et des pratiques d'un coutumier musical affaibli par la modernité ou en voie de disparition, mais encore la nouveauté de leur démarche par rupture de méthode, par déplacements et élargissement du champ d'investigation ${ }^{16}$. Le tout sous l'impulsion d'une ethnologie qui, en France légitimait les programmes de recherche, d'inventaire et de conservation des musées d'ethnographie. Les recherches et les collections musicales y seront homologuées à partir de 1955 sous le terme d'ethnomusicologie.

11 Un important changement allait progressivement s'imposer dès lors que les continuateurs d'un «folklore musical français » au Mnatp, au lendemain de la seconde guerre mondiale, voire dès le milieu des années 1930 purent bénéficier d'un statut stable de permanents, attachés à la documentation et à la collecte. La plupart de ceux qui, jusque là en France avaient concouru à la dynamique de collecte, avaient participé 
à dresser les contours d'une activité spécialisée qui n'était pas encore une "profession». Bien des noms importants dans ce concours, les Tiersot, Coirault et d'autres «s'adonnaient " sur leur «temps d'à-côté » à une telle activité de collecte, d'archivage et de consultation documentaire, relevant eux-mêmes de diverses professions, fréquemment de service public (bibliothécaires, instituteurs, inspecteurs d'académie, etc.) ou en en étant retraités. Mais l'exercice à plein temps, stable, permanent, rémunéré pour cette activité en tant que telle n'existait pas vraiment sinon exceptionnellement et de manière provisoire à travers l'octroi d'une " mission " ministérielle limitée dans le temps. La création du Musée des Arts et Traditions Populaires en 1937, à partir duquel de " petites mains » trouveront l'opportunité d'une stabilisation administrative et, progressivement, s'auto-délimiteront un profil de tâches jusqu'à en faire une spécialisation, marquera une étape importante dans l'entretien de la Geste dans la modernité : l'entrée en professionnalisation. C'est à partir de cette base, initiée dans l'espace (provisoire) du Palais de Chaillot au Trocadéro qu'allait peu à peu se définir la spécialisation programmatique qui prendrait nom "d'ethnomusicologie de la France " après d'autres intitulés comme ceux de musique folklorique ou de folklore musical; elle partagerait son plein temps entre « prospections systématiques » sur l'hexagone, archivages documentaires, publications monographiques ou méthodologiques, tribunes internationales, enseignements.

Derrière la continuité de carrières professionnelles, le programme d'ethnographie musicale ainsi homologué comme spécialité institutionnelle du Mnatp arriverait à un tournant décisif dans les années 1970. La période de ce que l'on y appelait les " prospections » ou " enquêtes systématiques » à l'intérieur de l'hexagone qui avaient permis de constituer l'essentiel des collections musicales, s'était achevée à quelques exceptions près vers la fin des années 1960. Cet achèvement coïnciderait approximativement avec l'installation du Mnatp dans ses nouveaux locaux attenants le Jardin d'acclimatation (1969). À partir de ce moment, ainsi stabilisée au sein de l'établissement du Mnatp en un périmètre architectural quasiment réservé à cet effet, la spécialisation musicologique, allégée de ses programmes de prospection hexagonale, pouvait s'appliquer à s'occuper d'elle-même, en l'état. La décennie 70 serait alors consacrée notamment à ce que les fondateurs appelaient une «exploitation systématique raisonnée » des collections musicales, sous le projet ancien de publication d'un Corpus de la musique traditionnelle française, devenu désormais Corpus des musiques orales de France et à l'instar des corpus publiés par le Mnatp - mais qui ne verra jamais le jour.

13 Dans ces mêmes années 1970, la classe d'objets qui avait été privilégiée dans les enquêtes systématiques et qui donnait lieu aux collections, n'intéressait pas seulement les spécialistes d'ethnomusicologie. De nouvelles générations de l'après guerre, influencées notamment par la folk music américaine s'en étaient saisies. Une importante dynamique de revival à travers concerts, disques, festivals, stages, clubs articulait cette mobilisation. Maintes fois sollicité pour ses collections sonores, le Mnatp ou du moins les responsables du Département d'ethnomusicologie - Phonothèque, resteraient obstinément à l'écart. Ce refus déclencherait des détestations à la hauteur de la passion qui animait la dynamique de revival. Dans l'esprit des fondateurs du Département, les collections patiemment établies par leurs soins constituaient un «matériau » spécialisé d'abord destiné à la recherche, qui ne pouvait être détaché d'un sens institutionnel propre à l'établissement et exigeant un appareil de scientificité qu'ils ne pouvaient être que les seuls à maîtriser. Les années qui suivront accentueront avec violence une 
coupure entre permanents théoriciens qui avaient formalisé leur propre profil de professionnalisation, et les héritiers de la grandeur d'un bénévolat d'investigation qui fut longtemps la marque prégnante de la Geste patrimoniale ethno-musicographique. Et qui ne pouvaient apparaître, face à des dispositifs et des normes mis en place par (et pour) les permanents, que comme autant «d'amateurs passionnés » - autant dire, peu distanciés au regard de l'exigence de détachement de la démarche de scientificité qui devait s'imposer?

14 Le monde des musiques traditionnelles qui allait alors s'organiser en ce début des années 80 rebattrait les cartes de la professionnalisation et des métiers de la Geste. La frontière établie unilatéralement entre professionnels théoriciens et "amateurs » ferait débat. Il pouvait y avoir des opportunités de professionnalisation autres que celle de théoricien ethnomusicologue; on pourrait, indépendamment de la filière disciplinaire, se doter de qualifications et de compétences en matière de "musiques traditionnelles » jusqu'à en faire un métier. Le volontarisme politique donnerait aux institutions de la culture la charge d'établir et de légitimer les compétences nécessaires à ces nouvelles qualifications : conservatoires ou écoles de musique, phonothèques ou médiathèques, centres de musiques traditionnelles, etc. Quelques uns de ces futurs professionnels étaient précisément auditeurs, boursiers ou libres, du présent séminaire.

\section{Dramatiques de l'irréversibilité - La médiation graphique}

15 «Les sons périssent parce qu'ils sont impossibles à écrire »: la formule d'Isidore de Séville, fut-elle fortement rabâchée, permettait d'introduire à l'une des grandes problématiques du séminaire, entrevue précédemment: la médiation graphique (système de notation musicale occidentale) et sa montée progressive, notamment quelques premiers essais ${ }^{17}$. Comment «faire tenir ", retenir un son, une parole? La question ne concernerait pas directement les équipements de garantie déployés par nos sociétés (cérémonialités de signature, serments, promesses et autres exceptionnalités réglées par des procédures d'engagement de la personne) pour faire "tenir (la) parole ", mais plutôt les formes procédurales d'arraisonnement physique d'une énonciation vocale. Comment capter, transporter des sons de voix, les conserver, indissociables qu'ils sont d'un lieu et de la présence physique qui les procurent à entendre, sans avancer d'emblée la solution moderne de l'enregistrement sonore? Quelles en ont été les premières formulations?

16 L'objectif de cette thématique, dans un contexte d'époque marqué en anthropologie par les travaux de Jack Goody sur la "raison graphique», était d'abord d'opérer un décentrement au regard de familiarités notamment solfégiques, en faisant un retour sommaire sur les premiers essais neumatiques qui ouvriront la voie à notre système de notation musicale. À quelles préoccupations répondaient ces premiers essais d'arraisonnement d'actions vocales? Comment imaginait-on sortir et désolidariser une production sonore de l'épaisseur des corps et des présences qui la procuraient à entendre, dans une culture - le christianisme et ses liturgies - aux prises avec une visualité du divin: techniques de schématisations visuelles comme enluminures de parchemins, miniatures, reliques enchâssées, etc. ? Ces questions permettaient chemin faisant d'insister sur la corporéité des énonciations et des pratiques, jusqu'à problématiser la question d'une "composante rhétorique " des actions musiciennes 
(ordre des techniques et pratiques d'exécution). Il ne s'agissait pas, non plus, de descendre dans l'érudition paléographique mais de s'interroger schématiquement sur un procédé d'anamorphose musicale, de conversion graphique qui va s'instaurer dans la mise au point visuelle, progressive, d'un abaque du sonore. Celui-ci fera notamment, s'articuler sur une portée à cinq lignes, des conditions ou des normes de mesure du temps (la durée, dans une linéarité de gauche à droite) et des hauteurs (une verticalité, de bas en haut), ouvrant sur une "naturalisation» de mécanismes ou de comportements musiciens souvent élémentaires comme ceux de représentation graphique, lecture, déchiffrage, audition mentale, etc. - ce que j'avais schématisé par l'expression de « raison musico-graphique ».

17 Pour saisir le déroulé de cette option d'objectivation et d'inscription graphique de la musique, du chant, il importait d'emblée de se départir d'une conception "naturalisée » de la ligne mélodique comme entité abstraite spécifiquement d'ordre musical et désolidarisée de tout texte, qu'un système de signes permet d'objectiver avec précision dans l'espace de la portée et qui est ainsi rendu disponible pour une prise en charge vocale et instrumentale à travers un acte lexique se donnant pour impératif une " conformité d'intégrité ${ }^{18}$ ». L'objectif du séminaire était de résumer, au prix d'une grande simplification, de surcroît en un domaine où les certitudes font facilement débat, comment s'était enclenchée la dynamique qui aboutirait à la ligne mélodique ainsi délocalisée d'un acte de chant et de sa corporéité, et plus tard au système actuel de notation graphique. Le contexte politique et religieux dans l'espace carolingien imposa à une vaste échelle la « romanisation » des liturgies chrétiennes et avec elle une homogénéisation des livres - les répertoires "grégoriens »-, nécessitait que, pour leur diffusion, l'on résolve la question pratique et urgente d'une reproductibilité de ces répertoires « rituellement » actualisés par des actions de chant de cadre liturgique. Cela impliquait aussi de schématiser les manières de réaliser ces actions vocales (psalmodie, lectionnes, chant) en les figurant visuellement sur les mots latins à prononcer. C'est sur la base d'une épaisseur vocale du texte que vont se négocier les premiers signes visuels schématisant des manières de conduire la voix, éventuellement doublés de gestes de la main (chironomie) dans la circonstance appropriée d'une cérémonie cultuelle. La matrice est celle du texte, de la phrase et de ses syllabes que doit faire lever la voix : des mots liés entre eux, un débit, des accentuations, une ponctuation et ses respirations, etc. ; au bout du compte, une expressivité. Aussi, c'est à l'instance grammaticale et ses signes graphiques (per notas) que recourent les premiers notateurs pour consigner le plus commodément l'action vocale à ( $\mathrm{re}$ ) produire. Les premiers essais se «bricolent " dans l'espace du parchemin ou de la page, au dessus du texte latin et entre deux lignes de texte, épousant ainsi une directionnalité de l'acte lexique, de gauche à droite. Il n'est pas encore question de fermeté intervallique, mais plutôt d'un mouvement vocal ascendant ou descendant qui se réalise sur des syllabes. La diastématie, mode de précision de la grandeur des intervalles, s'ébauchera progressivement vers le $\mathrm{x}^{\mathrm{e}}$ siècle à partir d'une ligne tracée au-dessus du texte, autour de laquelle seront étagées, audessus et au-dessous, des signes de plus en plus fermes. Bientôt s'ajouteront d'autres lignes, avec des précisions croissantes, y compris avec des jeux de couleurs... En somme, le souci des premiers notateurs semble concerner, in fine, l'action profératoire même, conduction vocale d'énoncés dans leur dimension « communicative »: l'allure sonore que doit présenter l'acte de chant.

18 C'est à partir de ce contexte, où pour l'heure on parle plus volontiers de voix (vox) que de sons musicaux, que vont s'accumuler progressivement les avancées d'une figuration 
graphique se donnant ainsi pour objet le mouvement continu de la voix dans la matière du texte. Telle sera la toile de fond à partir de laquelle va progressivement se développer, s'enrichir de trouvailles, se préciser et se théoriser une technique d'objectivation par mise à plat graphique et étalement dans l'espace de la page. Cette option de représentation graphique, partie du son vocal, va progressivement initier une dynamique d'objectivation graphique, spatio-visuelle du sonore qui, croisée avec d'autres conceptualisations ou théorisations, va installer la logique de centration et de resserrement sur le matériau sonore qui deviendra de plus en plus une abstraction une réification visuelle de la musique (la raison musico-graphique). Il y a loin assurément de ces premiers essais de détachement visuel d'une ligne mélodique, à notre système actuel d'objectivation graphique par notation musicale. Celui-ci deviendra un dispositif de contrôle de plus en plus serré de la communication musicale d'une œuvre en son dernier moment, son exécution - sa mise en audition. L'ethnomusicologie elle-même affinera le dispositif lorsque le magnétophone fera de l'étalement graphique à vue - des jeux d'écriture telles que les transcriptions synoptiques - une épreuve spécialisée de programmes de laboratoire ${ }^{19}$.

\section{«Composante rhétorique » de l'action musicienne}

19 L'ensemble des développements précédents proposant un regard distancié sur deux des modes d'arraisonnement sonore, par report (enregistrement magnétique, etc.) et par objectivation graphique (notation musicale) conduisaient implicitement à une autre problématique, d'ordre méthodologique: que retenons-nous dans nos descriptions, des actions constitutives de pratiques comme, par exemple, celles que nous appelons communément "chanter", "jouer" (d'un instrument de musique), "exécuter", etc.? Ou même "écouter»? Quel niveau d'organisation de ce qui s'accomplit retenir sous ces termes dans une culture, la nôtre, d'hyper-centration immédiate sur la production ou la matière sonore? Comment procède un corps, une présence physique pour " chanter »? Les actions à placer sous ce verbe inchoatif et leur coordination (maitriser son souffle, ouvrir la bouche, articuler, se tenir debout, se concentrer, procéder à un acte lexique, tourner une page, etc.) qui configure la pratique en tant que telle sont multiples, diverses: certaines, élémentaires, sont passées sous silence par nos descriptions tant elles nous semblent aller de soi, ou inutiles ou superflues au regard de la pertinence de notre objet, ou qu'elles sont indétectables. Généralement nous découpons la pratique en retenant (sélectionnant) ce qui nous paraît devoir constituer l'essentiel de signification de ce nous observons, en fonction du programme que nous nous sommes fixés et de l'horizon de nos évidences.

20 Le questionnement se proposait ainsi d'attirer l'attention sur la description du travail pratique de l'effectuation vive d'une action musicienne, le niveau d'individuation qu'en découpe l'observateur dans l'en-cours d'une "situation musicale». La posture de centration de l'observateur sur les opérations d'acheminement de sons en audition dans un particulier spatio-temporel (action située) sous-tendait diverses questions. Question par exemple de la "schématisation " visuelle - partition annotée, livre de chant plus ou moins bricolé, abstractions formulaires plus ou moins inscrites matériellement par graphisme ou autre, liste d'unités de base sur un bout de papier, etc. - qui permettait, ou d'où procédait le travail d'acheminement comme mise en perception visuelle et auditive dans un cadre et un espace donnés : question alors de la 
«forme » que prenait ce qui avait été pensé pour produire des effets (en tant qu'elle avait été préalablement pensée), l'épreuve qui la faisait aboutir perceptivement - ce qui en parvenait concrètement à un auditoire, réel ou fictif. Question également de l'auditoire, de la relation d'entendre (et sa spécialisation "écouter »), de son contact physique ouvrant sur des évaluations perceptives, des descriptions de la part des auditeurs.

Ces éléments seraient corrélés sous la problématique d'une " composante rhétorique " de l'action musicienne. La formulation suggérait une grille de lecture relativement commode référant notamment à l'une des grandes parties de notre ancienne rhétorique, l'Actio; elle permettait d'identifier $\mathrm{du}$ moins en toute première approximation avant de descendre dans les détails et les questionnements, l'articulation - les interactions - autour de ce que l'on vient d'évoquer sous le terme de "forme ", non seulement dans sa dimension possible (celle dessinée en creux par une schématisation), mais effective, en ce qu'elle parvient à un auditoire et produit des " effets». Autrement dit, la mise en contact comme moment de pratique et de la communication perceptive d'une «œuvre ». Ce poste de l'actio pouvait être ainsi celui de la présence même de l'acteur musicien: présentation de soi, tenue du corps, technicité en tant que procédure ostensive de fabrication et d'une présence (jusqu'à la virtuosité) qui se donne en visibilité avec le souci de son propre déploiement - de l'attitude corporelle jusqu'à l'habillement, en passant par la maitrise des gestes ou l'aménagement d'un environnement. Nos manuels ou méthodes d'apprentissage du chant ou d'un instrument de musique ne pouvaient-ils pas être interrogés dans cette perspective, comme autant de pages détachées ou inspirées de cette grande partie de notre ancienne rhétorique ? La pédagogie des traités consistera notamment à anticiper et gérer les tensions entre schèmes procéduraux à intérioriser (modalités d'attaque du son, souplesse du poignet, doigtés, bonne articulation de chevilles et croisements sécurisés des talons et pointes des pieds, etc.) qui constituent autant d'opérations techniques à effectuer, et une économie à la fois ergonomique et esthétique de "faire " (making): maîtrise des corps, de la fatigue musculaire et de la présence physique, inhérentes à une logique opératoire. Bref, une dimension incarnée et spectaculaire de la musique?

\section{Une anthropologie (pragmatique) de « la musique »?}

La conception " événementielle » du son qui traversait l'ensemble des problématiques précédentes amenait ainsi à cerner, dans le cadre d'un programme " d'ethnologie de la musique » de nos propres sociétés, quelques unes de nos évidences (essentialisations) en matière de musical. Si les développements de l'objectivation graphique ont pu progressivement instaurer une "réification visuelle» de la musique à travers la partition, ceux du transfert acousmatique de situations musicales comme avec le disque, contribueront à généraliser cette autre représentation - manipulation pratique - de la musique, d'une pièce musicale : une surface sonore mobile, autonome, univoque et portable, dotée du pouvoir prestigieux de défier les lois de la pesanteur. Libérée de tout encombrement physique des présences qui l'ont performée, n'importe quelle implémentation située, par exemple d'une partition de Beethoven ainsi transférée, semble pouvoir se tenir, se déployer d'elle-même dans l'espace sans jamais craindre les lois de la gravité - à la différence du support qui assure son transfert pour l'enrôler 
dans de nouveaux cadres d'espace-temps de présences et d'écoute: phonothèques, discothèques. N'était-ce pas l'une des grandes familiarités de notre modernité avec « la musique » en ses émergences sonores?

Les dernières années du séminaire ont été marquées par un retour sur quelques auteurs comme John Blacking, Marcel Mauss ${ }^{20}$. S'il devait y avoir quelque chose d'universel qui puisse mériter le nom de «musique » (M. Mauss) - pourquoi pas? -, encore fallait-il s'entendre sur ce que pourrait désigner « notre musique » ou " grammaire musicale »? Et si l'ethnomusicologie s'attachait à nous faire connaître ce que pouvait être cette "chose » dans les sociétés lointaines: qu'en était-il de l'ethnomusicologie régionale attachée à nos propres sociétés ? De l'ethnomusicologie de la France par exemple, qu'en connaissions-nous, sinon celle se donnant pour objet les «musiques traditionnelles »programme d'une ethnomusicologie inventoriale qui ne semblait pas "épuiser » ni même redouter la question maussienne de « notre musique " ? Certes, nous avions aussi la musicologie - « espèce particulière de musique ethnique » (J. Blacking) ? Épuisait-elle elle-même ce qui restait de "musique » non traité par l'ethnomusicologie régionale ("de la France») telle que nous la connaissions? D'autres secteurs disciplinaires, notamment la sociologie, travaillaient également en ce domaine ${ }^{21}$. Il ne resterait plus alors qu'à procéder par assemblage des secteurs pour avoir quelque idée de ce que pourrait être «notre musique » ? Étonnant exercice aporétique.

Le cadre anthropologique s'avérait plus pertinent - autant dire, accueillant. Pourquoi pas « la-musique », et pour commencer « notre musique » comme objet empirique, par delà les grands partages disciplinaires (secteur de la musique savante, écrite, pour les uns, secteurs des musiques orales pour les autres) ? En résumé, un programme à l'instar de celui de l'ethnomusicologie lorsqu'elle se donne d'étudier la dynamique organisatrice et instituante d'un «musical» dans des sociétés que l'on disait par commodité « lointaines » ou " autres », pourrait-il avoir quelque pertinence rapporté à nos propres sociétés qui disposaient déjà d'un vaste domaine spécialisé d'investigations se donnant le « musical » comme objet de descriptions ? Ces descriptions permettaientelles de répondre à la question sous-jacente du programme : comment nos sociétés s'y prennent-elles pour faire exister la chose, la tenir en évidence? Deux grandes hypothèses se proposaient alors d'expérimenter dans un premier temps le questionnement. La première posait que ce que nous considérions sous le vocable de "musique « et dans la catégorie générique du même nom devait être le résultat de ce que nos sociétés y inscrivaient. Il n'était de « musique » hors d'un « faire » multiforme (to make; to do): hors des activités et dispositifs multiples qui se donnaient mutuellement appui pour réaliser les choses, que ce soit par exemple dans la confection, l'anticipation, l'accomplissement d'effectuations musiciennes (" composer", " exécuter", "interpréter", etc.). Dans l'audition et ses niveaux d'attention, dans les manières d'en parler, de rendre compte et de qualifier. Dans les lieux, espaces, monuments, objets ou ustensiles divers dont nous nous servons, que nous affectons ou consacrons à la «chose ". Seconde hypothèse, corollaire de la précédente : nous nous mouvons en permanence, s'agissant de nos sociétés, dans une conception de "Musique », compromis ou tension entre un idéal d'immatérialité et la corporéité qui la fait émerger en tangibilité, dans l'opacité et la pesanteur des présences déployées dans l'univers de la dimensionnalité et de l'orientation, de l'étendue et du mouvement. La "Musique-avec-un-grand-M ", disait-on familièrement comme d'une entité universelle, ineffable, transcendante, etc. 

constituer un programme d'anthropologie (pragmatique) de la musique. Il fallait commencer, pour voir. C'était alors la dernière année du séminaire (1993) ${ }^{22}$. Les décentrements qu'imposait ce programme nécessitaient des moyens théoriques et descriptifs d'autant plus coûteux qu'il fallait dépenser une importante énergie sinon pour convaincre du moins pour sensibiliser quelques "appareils de discernement " parfois décontenancés, s'obstinant à solliciter l'avis d'experts ethnomusicologues... qui, encore dans les années 1990, considéraient un tel programme comme superfétatoire face à l'urgence de collecter ce qui pouvait encore rester de «vraie musique traditionnelle » sur l'hexagone...

Je reprendrai ultérieurement ce programme avec d'autres, collègues et doctorants, à Marseille dans le cadre du Centre Norbert Elias (ex-Shadyc), notamment autour de la question des catégorisations musicales, haut lieu de relations entre théorisations multiples et pratiques ordinaires - des «théories musicales ordinaires ${ }^{23}$ ». Le music-hall y serait un genre « bon à penser »...

\section{BIBLIOGRAPHIE}

BECKER, Howard S., Les mondes de l'art, Paris, Flammarion, 1988, 2010.

BLACKING, John, Le sens musical, Paris, Minuit, 1980.

CERTEAU, Michel de, L'écriture de l'histoire, Paris, Gallimard, 1975.

CERTEAU, Michel de, La culture au pluriel, Paris, UGE, 1974.

CHEYRONNAUD, Jacques « Ethnologie et musique. La question de l'objet », Ethnologie française, 3 (1997), p. 382-393.

CHEYRONNAUD, Jacques, « Une vie consacrée à l'ethnomusicologie. Claudie Marcel-Dubois (1913-1989) », Cahiers des musiques traditionnelles 3 (1990), p. 173-179.

CHEYRONNAUD, Jacques, Instructions pour un Recueil général des poésies populaires de la France. L'enquête Fortoul (1853-1857), Paris, Comité des Travaux historiques et scientifiques, 1997.

CHEYRONNAUD, Jacques, Mémoires en recueils. Jalons pour une histoire des collectes musicales en terrain français, Montpellier, Office départemental d'action culturelle, 1986.

CHEYRONNAUD, Jacques, Musique, politique, religion. De quelques menus objets de culture, préface de Jean-Louis FABIANI, Paris, L'Harmattan, 2002.

COULANGEON, Philippe, «Les musiciens de jazz : les chemins de la professionnalisation », Genèse, 36 (1999), p. 63-64.

DUCHET, Marie-Élisabeth, « Description grammaticale et description arithmétique des phénomènes musicaux : le tournant du Ix ${ }^{\mathrm{e}}$ siècle », Sprache und Erkenntis im Mittelaiter, Miscellanea Medievalia XIII/2 (1981), p. 561-579 
DUFOURT, Hugues, « L'artifice d'écriture dans la musique occidentale », Critique 408 (1981), p. 465-477.

FONTAINE, Jacques, Isidore de Séville et la culture classique dans l'Espagne wisigothique, Paris, Études augustiniennes, 1983 (3 volumes).

GOODY, Jack, La raison graphique. La domestication de la pensée sauvage, traduit de l'anglais et présenté par Jean BAZIN et Alban BENSA, Paris, Éditions de Minuit, 1979.

HAMELINE, Jean-Yves, « Le chant Grégorien », Histoire de la musique. La musique occidentale du Moyen Age à nos jours, sous la direction de Marie-Claire BELTRANDO-PATIER, Paris, Bordas, 1982, p. 19-46.

HENNION, Antoine, Comment la musique vient aux enfants. Une anthropologie de l'enseignement musical, Paris, Anthropos, 1988.

HENNION, Antoine, La passion musicale. Une sociologie de la médiation, Paris, Métailié, 1993.

LATOUR, Bruno, « Les “vues” de l'esprit », Culture technique 14 (1985), p. 5-29.

LENCLUD, Gérard, « En être ou ne pas en être. L'Anthropologie sociale et des sociétés complexes », L'Homme 97 (1986), p. 143-153.

LENCLUD, Gérard, « La tradition n'est plus ce qu'elle était ... Sur les notions de tradition et de société traditionnelle », Terrain 9 (1987), p. 110-123.

MAUSS, Marcel, Cuvres. 2. Représentations collectives et diversité des civilisations, Paris, Minuit, 1969.

PEDLER, Emmanuel et CHEYRONNAUD, Jacques (eds), « Théories ordinaires », Enquête 10 (2013).

\section{NOTES}

1. Qui était l'initiatrice, avec Georges Henri Rivière, de cette branche régionale dite «Ethnomusicologie de la France» qui se déploiera sous la tutelle du Musée national des Arts et Traditions populaires (désormais Mnatp) au Palais de Chaillot puis à Neuilly près du Jardin d'acclimatation à partir de 1969. Voir CHEYRONNAUD, Jacques, Musique, politique, religion. De quelques menus objets de culture, préface de Jean-Louis FABIANI, Paris, L'Harmattan, 2002, p. 157-197 ; également : CHEYRONNAUD, Jacques, « Une vie consacrée à l'ethnomusicologie. Claudie Marcel-Dubois (1913-1989) », Cahiers des musiques traditionnelles 3 (1990), p. 173-179.

2. Voir sur ce point LENCLUD, Gérard, «En être ou ne pas en être. L'Anthropologie sociale et des sociétés complexes », L’Homme 97 (1986), p. 143-153.

3. Un volume de la revue Ethnologie française $(3,1984)$ réunit des articles des doctorants et étudiants du séminaire de $\mathrm{M}$. Andral à l'époque, sous la question des "nouveaux objets » en « ethnomusicologie de la France ». Cl. Marcel-Dubois en écrivit une conclusion.

4. La suggestion émanait d'Isaac Chiva (1925-2012), Directeur d'études et l'un des principaux initiateurs de la Mission du patrimoine ethnologique. Le programme d'enseignement que je présentai s'inspirait de grandes lignes de mon projet de recherche $\mathrm{Cnrs}$ en anthropologie. Comme toute construction rétrospective, la relecture proposée ici n'échappera sans doute pas à quelques ajustements postérieurs ; toutefois, j'ai tenté de reconstituer au mieux, avec une certaine liberté de ton, ce qu'il pouvait en être à l'époque. Voir également, en complément actualisé du présent article : Jacques CHEYRONNAUD, «Ethnomusicologie de la France et Mission du Patrimoine ethnologique au tournant des années 1980. Une brève collaboration », Noël BARBE et 
Jean-Louis TORNATORE (eds), Actes du colloque «Le moment du Patrimoine ethnologique ", Dijon, décembre 2016 (à paraître).

5. Le terme ne désignait pas tout à fait le même profil dans les deux cas. Les deux tutelles de l'établissement musée-laboratoire Mnatp/Centre d'Ethnologie française (Direction des Musées de France et Cnrs) laissaient à cet établissement l'initiative de son organigramme interne, et ne reconnaissaient d'autre interlocuteur que sa direction. Si bien que la gestion administrative (locaux, personnels, crédits de fonctionnement, etc.) du Département-Phonothèque était déléguée avec bienveillance par la direction du musée-laboratoire qui donnait ou non son accord de principe - cette autonomie de gestion toute relative relevait surtout d'un rapport de force, rarement défavorable, avec la direction de l'établissement. La seule autonomie possible en droit était donc essentiellement programmatique.

6. Inspirée des «mondes de l'art» (BECKER, Howard S., Les mondes de l'art, Paris, Flammarion, 1988, 2010), la formule "monde» (sous-entendu ici : des musiques traditionnelles ») renvoie à cette réalité complexe en ce début des années 80 de rapports entre des acteurs multiples, individuels ou collectifs, privés ou publics (organisations associatives, administrations centrales ou régionales, établissements publics, maisons d'édition, etc.) à propos de savoirs et de pratiques concernant la classe spécifique d'objets en question sous le label « musiques traditionnelles ».

7. J'emprunte la formule à : COULANGEON, Philippe, «Les musiciens de jazz: les chemins de la professionnalisation", Genèse 36 (1999), p. 63-64. C'est-à-dire ici : qui devrait passer préalablement par la maîtrise des signes de notation graphique, donnant ainsi primauté à l'acte lexique de conformité d'exactitude ou d'intégrité (cf. infra, note 15) et aux règles solfégiques dans tout apprentissage délivrant une compétence professionnelle telle qu'elle pouvait être homologuée en premier lieu par les conservatoires.

8. Voir LENCLUD, Gérard, « La tradition n'est plus ce qu'elle était ... Sur les notions de tradition et de société traditionnelle», Terrain 9 (1987), p. 110-123. L'article reprenait une conférence de l'auteur prononcée à l'ouverture d'une session de formation organisée par la Mission du Patrimoine à Saint-Malo en 1986.

9. Il ne s'agissait pas d'enseigner directement l'ethnomusicologie, ni de doubler l'enseignement dispensé à Paris X ; l'intitulé Ethnomusicologie visait à offrir la possibilité d'une initiation aux protocoles d'enquête et d'analyse dans des séances libres, ouvertes à tout public au Département d'ethnomusicologie du Mnatp une fois par semaine; par ailleurs, une initiation à «l'enquête ethnomusicologique " dans le cadre du DEA en ethnologie était également dispensée par le Lacito, sous l'autorité de Simha Arom.

10. Comme ceux, par exemple de "chants et hymnes de la Révolution ", "chant scolaire", "chant grégorien", «chant populaire», etc. Le terme "hymnodie» visait à problématiser diverses questions sous-jacentes aux projets de regroupements d'énoncés et/ou de ressources musicales en répertoires par affiliations formelles, thématiques ou fonctionnelles, d'enrôlements institutionnels au service de l'entretien de croyances ou de convictions et de célébration d'un "Commun idéal » (ce qui permet à un collectif, dans son programme d'exigences et de finalités, de s'éprouver et de s'exposer comme " unitaire » autour d'une célébration commune d'entités ou de valeurs). Dans cette même perspective, la parodie polémique (anticléricale ou autre «anti») empruntant à des répertoires adverses pouvait s'analyser comme relevant d'un fonctionnement normal des "clôtures", tant pour désigner une altérité plus ou moins menaçante que pour annuler et s'approprier des énoncés adverses?

11. Cf. infra. La parution française en 1979 de l'ouvrage de Jack Goody (La raison graphique. La domestication de la pensée sauvage. Traduit de l'anglais et présenté par Jean Bazin et Alban Bensa, Paris, Editions de Minuit, 1979), et les enseignements notamment du musicologue théologien Jean-Yves Hameline, de Jean Bihan en sémiologie grégorienne et du sémiologue italien Gino Stefani à l'Institut de musicologie de l'Institut Catholique de Paris au début des années 1970 seront pour beaucoup dans l'élaboration de cette perspective ; voir également ci-dessous. 
12. Voir CHEYRONNAUD, Jacques, Mémoires en recueils. Jalons pour une histoire des collectes musicales en terrain français, Montpellier, Office départemental d'action culturelle, 1986.

13. Sur le cadre de construction de cette problématique à l'époque (y compris la conception événementielle du son qui peut lui être sous-jacente), cf. : CERTEAU, Michel de, La culture au pluriel, Paris, UGE, 1974, en particulier p. 55-94; également: CERTEAU, Michel de, L'écriture de l'histoire, Paris, Gallimard, 1975, p. 215-248 ; également LATOUR, Bruno, «Les “vues" de l'esprit », Culture technique, 14 (1985), p. 5-29. Cf. également infra.

14. Cf. CHEYRONNAUD, Jacques, Instructions pour un Recueil général des poésies populaires de la France. L'enquête Fortoul (1853-1857), Paris, Comité des Travaux historiques et scientifiques, 1997.

15. Cf. CHEYRONNAUD, Mémoires en recueil, op. cit., 1986.

16. Notamment avec l'intérêt organologique et plus globalement, sous l'appellation générique de "faits musicaux », avec un élargissement aux " phénomènes de musique » et de " paramusique », des instruments de musique aux « engins sonores ». Voir CHEYRONNAUD, Musique, politique, op. cit., p. 157-197. « Ces buts, écrira Cl. Marcel-Dubois, /.../ cadrent très exactement avec ceux de ma discipline » l'ethnomusicologie. Voir MARCEL-DUBOIS, Cl., Rapport de conjoncture, $1^{\text {re }}$ partie, § 3, février 1964, p. 5 (reproduction communiquée à l'auteur par Maguy P. Andral en 1987).

17. Parmi les travaux utilisés à cette occasion, outre les histoires de la notation musicale occidentale ou ma propre thèse sur les chantres d'église et les auteurs cités notes 10 et 11 : DUCHET, Marie-Élisabeth, «Description grammaticale et description arithmétique des phénomènes musicaux : le tournant du $\mathrm{IX}^{\mathrm{e}}$ siècle », Sprache und Erkenntis im Mittelaiter, Miscellanea Medievalia XIII/2 (1981), p. 561-579; DUFOURT, Hugues, "L'artifice d'écriture dans la musique occidentale », Critique 408 (1981), p. 465-477 ; FONTAINE, Jacques, Isidore de Séville et la culture classique dans l'Espagne wisigothique, Paris, Études augustiniennes, 1983 (3 volumes) ; HAMELINE, Jean-Yves, «Le chant Grégorien », Histoire de la musique. La musique occidentale du Moyen Age à nos jours, sous la direction de Marie-Claire BELTRANDO-PATIER, Paris, Bordas, 1982, p. 19-46. Je dois beaucoup à mes échanges avec J.-Y. Hameline alors qu'il préparait l'article précédent au début des années 1980.

18. Ma thèse sur les chantres d'église aux $\mathrm{XIX}^{\mathrm{e}}$ et $\mathrm{xx}^{\mathrm{e}}$ siècle faisait apparaître un autre type de conformité que je proposais d'appeler « conformité d'usage ». Il peut exister dans l'acte lexique, en ce qu'il consiste à tirer parti des ressources d'une schématisation graphique à partir de l'œil, une réalisation de "convenance" comme dans le cas de la notation carrée d'anciens livres de chœur, qui repose sur une mémoire procédurale «bricolant » divers accommodements tant avec la notation que le livre et la fonction. Ces bricolages mis au point par des chantres qui possédaient une formation «rudimentaire » ou parfois inexistante au plain-chant et au latin pouvaient se transmettre aux successeurs dans la fonction. Ces accommodements étaient rendus possibles dans un régime de familiarité attaché au cadre cultuel et à la fonction et son épais livre de chant spécialisé : caractères d'imprimerie, couleurs d'encre, signes divers dans le corps graphique, aménagements personnels dans le livre, occasions récurrentes (calendrier liturgique) engendrant des automatismes, lecture pictographique de la notation carrée, etc., produisaient en se donnant mutuellement appui ce type de conformité.

19. L'aménagement du dispositif notationnel musical sous le terme de "signes spéciaux » sera l'une des premières grandes préoccupations, internationale et consensuelle, dans les années d'après-guerre. Plusieurs réunions d'experts "sur la notation de la musique folklorique " organisées sous les auspices du Conseil international de la musique (Unesco) par les Archives internationales de Musique populaire se tiendront en 1949 et 1950 à Genève et à Paris. Voir Conseil international de la musique, Notation de la musique folklorique. Recommandations du Comité d'experts /.../, UNESCO, 1952.

20. En particulier : "Notre musique n'est qu'une musique. Et cependant, il existe quelque chose qui mérite le nom de "la musique". Ce n'est pas celle de notre "grammaire musicale", mais celleci y entre» (MAUSS, Marcel, CEuvres. 2. Représentations collectives et diversité des civilisations, Paris, 
Minuit, 1969, p. 152.) André Schaeffner, l'une des grandes figures initiatrices de l'ethnomusicologie en France, jouera un rôle important auprès de Mauss pour les questions musicales. Soit encore: "L'ethnomusicologie est en mesure de causer une révolution dans le domaine de la musique et de l'éducation musicale si elle va jusqu'au bout de ce qu'impliquent ces découvertes et si elle se constitue en méthode - et non pas tout simplement en secteur »; soit encore: "Il faut nous souvenir que dans la plupart des conservatoires on n'enseigne qu'une espèce particulière de musique ethnique, et que la musicologie est en réalité une musicologie ethnique » (BLACKING, John, Le sens musical, Paris, Minuit, 1980, p. 11-12.)

21. Notamment dans ces années 1980, la sociologie de la médiation au Centre de sociologie de l'innovation (CSI). Bien des questionnements au centre du séminaire recoupaient alors ceux d'Antoine Hennion. Cf. pour l'époque : HENNION, Antoine, Comment la musique vient aux enfants. Une anthropologie de l'enseignement musical, Paris, Anthropos, 1988, et bien évidemment, du même : La passion musicale. Une sociologie de la médiation, Paris, Métailié, 1993 (1 ${ }^{\text {re }}$ édition).

22. Les questionnements seront repris dans l'article: CHEYRONNAUD, Jacques «Ethnologie et musique. La question de l'objet ", Ethnologie française, 3 (1997), p. 382-393. Voir également leur actualisation dans CHEYRONNAUD, Musique, politique, op. cit., 2002. Je remercie vivement JeanLouis Fabiani pour son soutien intellectuel et amical durant bien plus que les quelques années qui suivront mon départ du Mnatp.

23. Voir PEDLER, Emmanuel et CHEYRONNAUD, Jacques (eds), « Théories ordinaires », Enquête 10 (2013).

\section{RÉSUMÉS}

L'article fait retour sur un séminaire qui, à l'époque, travaillait à formuler dans le cadre de l'anthropologie un objet "musique », à distance des orientations d'alors d'une ethnomusicologie régionale «de la France» telle qu'elle s'est institutionnalisée dans l'un des musées français d'ethnologie, le Musée national des Arts et Traditions populaires. L'article propose, chemin faisant, de traverser les décennies 1970-1980 et le tournant que devra prendre ce secteur disciplinaire.

\section{INDEX}

Mots-clés : ethnomusicologie de la France, musiques traditionnelles, pragmatique de la musique, anthropologie de la musique, action musicienne, notation musicale, enregistrement sonore, patrimoine musical

\section{AUTEUR}

\section{JACQUES CHEYRONNAUD}

Jacques Cheyronnaud (Limoges, 1949) Hdr. Chercheur au Musée national des Arts et Traditions populaires - Centre d'Ethnologie française, de 1973 à 1993. Directeur du Département 
d'Ethnomusicologie de la même institution, de 1987 à 1995. Directeur de recherche CNRS au Centre Norbert Elias (Marseille) et Directeur de thèses à l'EHESS (en retraite depuis 2014). 